“Transfer” XV: 1-2 (2020), pp. 207-232. ISSN: 1886-554

\title{
LA TRADUCCIÓN HOY EN DÍA: \\ RETRATO DE UNA PROFESIÓN FEMINIZADA. ASPECTOS ÉTICOS Y LABORALES
}

José Santaemilia (ORCID: 0000-0002-2845-5483)

Universitat de València

\section{La mujer en la universidad y en el mercado laboral: diagnóstico inicial}

Este artículo aportará datos para un debate sobre la presencia (y significación) de las mujeres en el mundo de la traducción profesional, sobre todo en la traducción literaria o editorial. Para ello, comenzaré observando qué presencia tienen las mujeres en la universidad y en el mercado laboral, atendiendo a las diversas disciplinas y ámbitos profesionales. Se trata de aportar datos para un debate en paralelo: por un lado, la mayor o menor presencia de mujeres en una disciplina o profesión como la traducción y, por otro lado, sobre la propia traducción en tanto que ámbito femenino -o feminizado-, como algunos estudios han sugerido.

Actualmente, en el estado español, las mujeres superan ligeramente a los hombres en número, pues representan el 50,96\% de la población, según los datos del INE ${ }^{1}$ para 2018. En el mundo de la enseñanza universitaria, desde el 8 de marzo de 1910, en que se permitió oficialmente a las mujeres matricularse en la universidad, hasta la actualidad, la cifra no ha dejado de aumentar. De hecho, desde 1986, las mujeres son mayoría en las universidades del estado, pues representaban el 50,1\% del estudiantado, siendo en 2010 el 54\% (véase Peña 2010). Según datos oficiales del Ministerio de Educación y Formación Profesional, ${ }^{2}$ durante el curso académico 2017-2018, el porcentaje de mujeres matriculadas en

\footnotetext{
${ }^{1}$ Disponible en: <<https://bit.ly/2kQqmYu>> (Última consulta: 14 de marzo de 2019).

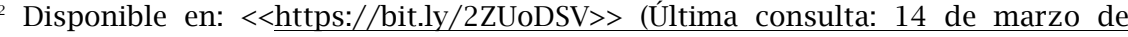
2019).
} 
“Transfer” XV: 1-2 (2020), pp. 207-232. ISSN: 1886-554

títulos de grado representaba el 54\%, en títulos de Máster el $54,76 \%$, mientras que en estudios de doctorado las mujeres suponen el 49,76\%. Además, las mujeres tienen (como promedio) más formación que los hombres: según los datos oficiales del Ministerio de Educación y Formación Profesional, un 39,8\% de mujeres cursa estudios superiores, mientras que el porcentaje de hombres se sitúa en un $34,6 \%$.

Si miramos los datos relativos a las principales ramas del conocimiento (Fig. 1), constatamos una (en ocasiones) preocupante brecha de género: por un lado, cuatro ámbitos claramente feminizados (Salud y servicios sociales, con un $80,82 \%$ de mujeres; Educación con un 76,95\%; Ciencias sociales, periodismo y documentación, con un 61,37\%; y Artes y Humanidades, con un 60,85\%), y por otro lado, dos ámbitos extremadamente masculinizados ${ }^{3}$ (Informática, con un exiguo 12,18\% de mujeres en las aulas; e Ingeniería, Industria y Construcción, con un 28,41\%). En medio quedan disciplinas con un porcentaje casi idéntico de mujeres y hombres (Negocios, administración y derecho; Agricultura, ganadería, silvicultura, pesca y veterinaria; y Ciencias) y finalmente Servicios (que estudian un $43,10 \%$ de mujeres).

Aunque se observa una presencia creciente de las mujeres en ámbitos en que apenas destacaban hace unas décadas, seguimos encontrando espacios profesionales refractarios a la presencia femenina. Dichos espacios giran en torno a temas tan relevantes para el progreso económico como la informática y las nuevas tecnologías, las ingenierías o la construcción.

\begin{tabular}{|l|l|}
\hline & Mujeres (\%) \\
\hline 01.-Educación & 76,95 \\
\hline 02.-Artes y humanidades & 60,85 \\
\hline $\begin{array}{l}\text { 03.-Ciencias sociales, periodismo y } \\
\text { documentación }\end{array}$ & 61,37 \\
\hline
\end{tabular}

3 Utilizo los términos feminizado y masculinizado como meros adjetivos calificativos, que hacen referencia al sexo de las personas. Más adelante incidiré en los aspectos cualitativos e ideológicos. 
“Transfer” XV: 1-2 (2020), pp. 207-232. ISSN: 1886-554

\begin{tabular}{|l|l|}
\hline 04.- Negocios, administración y derecho & 53,12 \\
\hline 05.- Ciencias & 49 \\
\hline 06.- Informática & 12,18 \\
\hline 07.- Ingeniería, industria y construcción & 28,41 \\
\hline $\begin{array}{l}\text { 08.- Agricultura, ganadería, silvicultura, pesca y } \\
\text { veterinaria }\end{array}$ & 51,95 \\
\hline 09.- Salud y servicios sociales & 80,82 \\
\hline 10.- Servicios & 43,10 \\
\hline
\end{tabular}

Fig. 1. Porcentajes de mujeres en la universidad española, según las diversas ramas del conocimiento (Datos de 2018, según el Ministerio de Educación y Formación Profesional).

Para completar esta visión del mundo de la universidad, constatamos la persistencia del llamado techo de cristal en el ámbito profesional: mientras las estudiantes representan el 54\% de su colectivo, el conjunto de profesoras supone el 40,9\% del total, según datos del Ministerio de Educación, Cultura y Deporte para el curso académico 2015-2016. Esta cifra contrasta de manera evidente con la educación infantil, ámbito en el que el 97,6\% del profesorado son mujeres. Dentro de las categorías docentes universitarias, constatamos (en la Fig. 2) que mientras las profesoras contratadas suponen el $44,3 \%$ del total, las profesoras titulares son un 39,9\%, y el porcentaje de catedráticas desciende al 20,9\%. En cuanto al número de rectoras, la elección de $\mathrm{M}^{\mathrm{a}}$ Vicenta Mestre como rectora de la Universitat de València ha elevado a cinco el número total de máximas dirigentes de universidades públicas del estado, lo que supone un 10\%. Los datos hablan por sí solos de un desequilibrio jerarquizado e institucionalizado. 
“Transfer” XV: 1-2 (2020), pp. 207-232. ISSN: 1886-554

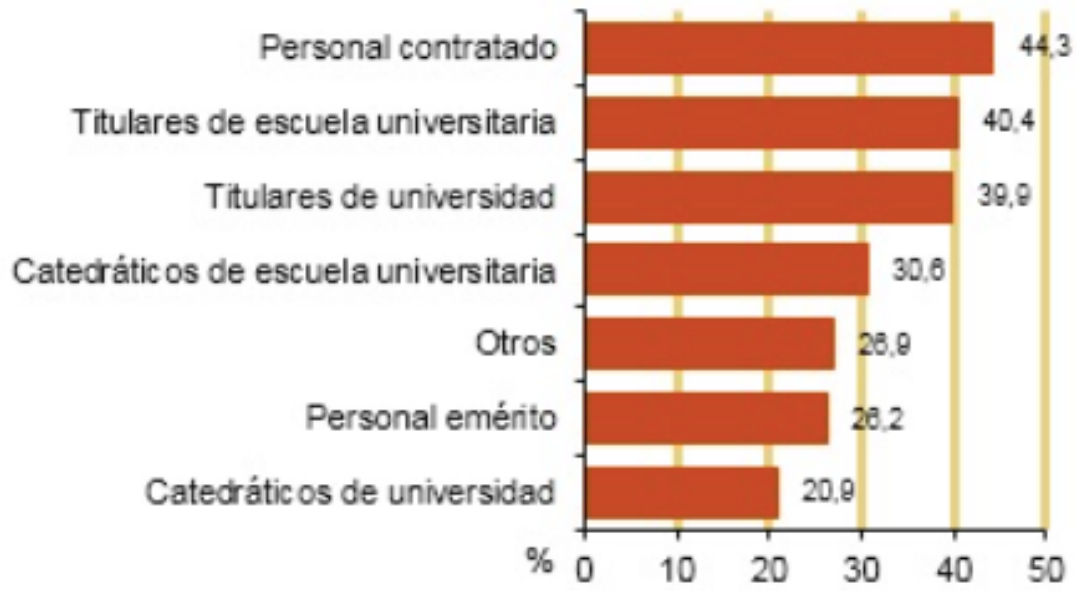

Fig. 2. Porcentaje de profesoras en las universidades españolas (Datos correspondientes al curso 2015-2016, según datos del Ministerio de Educación, Cultura y Deporte).

Cuando llegamos al mundo laboral (véase Fig. 3), ese caudal femenino, ese mayor porcentaje de mujeres que poseen (en conjunto) una mayor formación que los hombres, queda desdibujado y diluido en una fuerza de trabajo en que predominan claramente los hombres $(71,5 \%)$ frente a las mujeres $(59,6 \%)$. La cifra de estas últimas queda muy lejos de las expectativas de la Estrategia Europea 2020, que sitúa en un $75 \%$ la tasa de empleo, tanto de hombres como de mujeres, que deberían incorporarse al mercado de trabajo. Esto sitúa la brecha de género (en datos de 2017) en España en el mercado laboral en un 10,8\%, un dato ligeramente superior al de la Unión Europea (véase Fig. 4). Esta brecha, aunque va suavizándose a lo largo de las últimas décadas, sigue reflejando una inquietante tendencia: las mujeres están, mayoritariamente, en la base de la educación superior pero, a medida que crecen las exigencias y las responsabilidades de los puestos de trabajo, van desapareciendo paulatinamente y dejan paso a los hombres, cuya 
“Transfer” XV: 1-2 (2020), pp. 207-232. ISSN: 1886-554

formación de partida es inferior. En las siguientes páginas intentaremos resumir los argumentos de esta disparidad histórica.

Tasa de empleo según grupos de edad. 2017

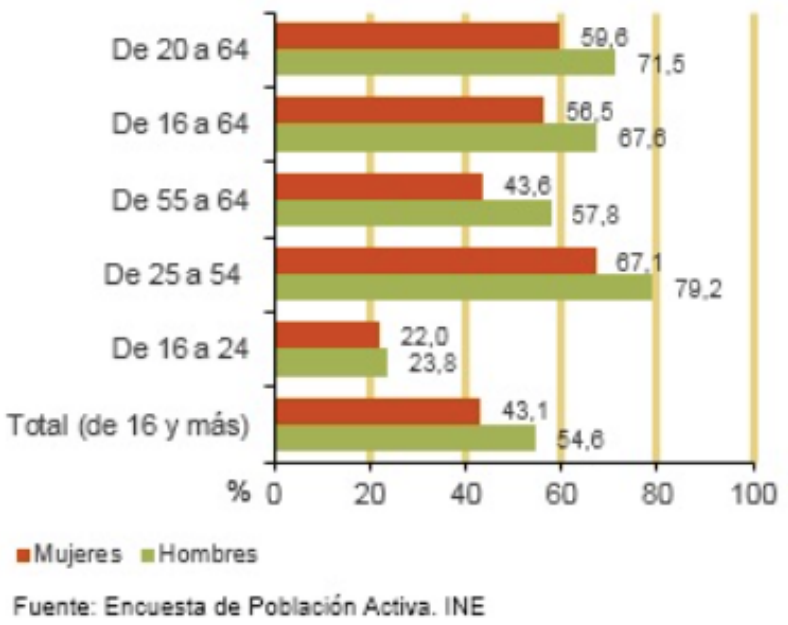

Fig. 3. Tasa de empleo comparada entre mujeres y hombres.

Universos plurales, cultura laboral singular. La mujer ante una cultura laboral hostil

De manera breve, señalaré a continuación los rasgos básicos del mercado laboral, tratando de proporcionar un diagnóstico básico del mismo, prestando atención a una perspectiva de género, al (des)equilibrio que se observa entre hombres y mujeres. 
“Transfer” XV: 1-2 (2020), pp. 207-232. ISSN: 1886-554

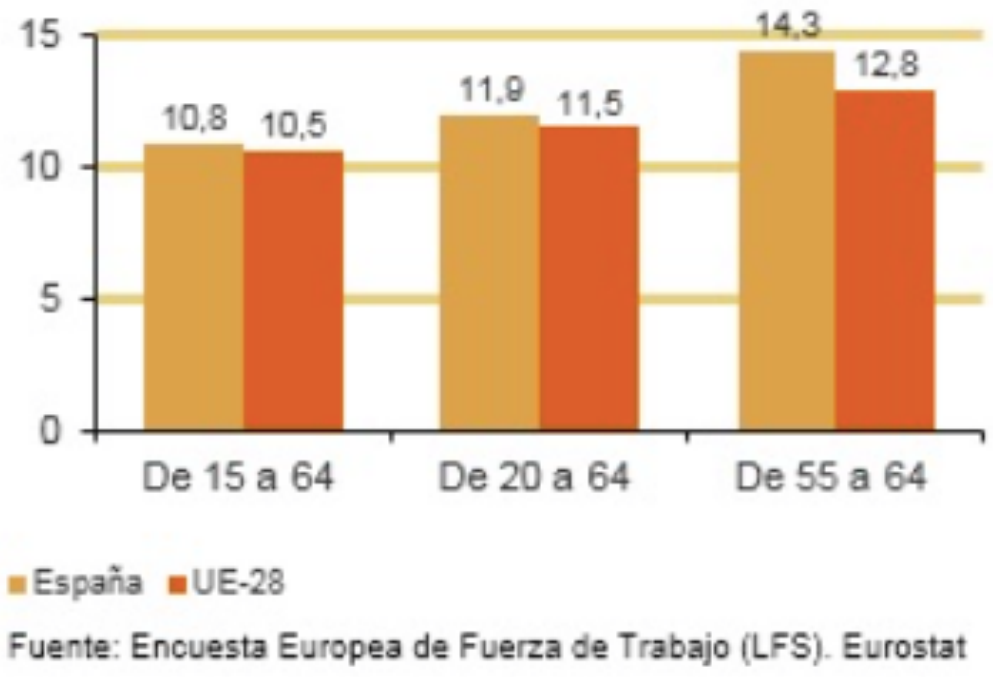

Fig. 4. Brecha de género en el mercado laboral (España vs Unión Europea)

\section{Existencia de una cultura laboral masculinizada}

Un rasgo primordial del mercado del trabajo lo constituye la existencia -ya tradicional, aunque suavizada recientemente- de una cultura laboral masculinizada. En este caso, damos a este adjetivo un valor no solo cuantitativo sino también cualitativo, al observar en el mundo del trabajo tendencias y rutinas que favorecen de manera clara a los hombres y se ajustan a sus ritmos biológicos y sociales. Ibáñez Pascual (2008: 89) habla de "la nueva lógica semipatriarcal de proveedor principal y proveedora secundaria (frente al modelo de varón proveedor y mujer cuidadora)." Las manifestaciones externas del fenómeno pueden cambiar pero, en esencia, se trata de variaciones sobre el mismo tema: las mujeres, en general, siguen siendo las candidatas más firmes a ocupar espacios de subordinación en la ecología laboral de nuestros días. Parece observarse que el género (esa construcción social que toma como base el sexo biológico y sobre la que se asientan 
“Transfer” XV: 1-2 (2020), pp. 207-232. ISSN: 1886-554

los prejuicios y los estereotipos) sigue presidiendo las relaciones sociales en nuestra sociedad y es, también, "un elemento estructurante fundamental de nuestro mercado de trabajo [...], pues los puestos de trabajo están sexuados, no son neutros." (ibidem: 117)

Esta cultura laboral masculinizada gira de manera capital en torno a los estereotipos de género, que Talbot define así:

the term 'stereotype' is often used to refer to prescriptions of behaviour or unstated expectations of behaviour, rather than specifically to representational practices. Gender stereotypes are closely linked with and support gender ideologies. (Talbot 2003: 30-31)

Dichos estereotipos, al estar ligados a ideologías de género, se perpetúan de manera sencilla y natural y acaban generando (y cimentando) las expectativas sociales en torno a la mujer, y nos llevan a dar por sentado -entre muchas otras cosas- que las muje-res asumirán mayores responsabilidades en el cuidado de la familia.

Se trata, en efecto, de una cultura de trabajo que, entre otras cosas, prescribe largas jornadas de trabajo; que premia la antigüedad (una herencia de la tradición, inequívocamente masculina) frente a la juventud o a la innovación; que minusvalora las cualidades que tradicionalmente se han considerado como 'femeninas'; y que genera un "mercado laboral dicotómico" (Antonio Villar, cit. en Tobarra 2010) que constituye un elemento de discriminación hacia las mujeres, al proteger a los trabajadores fijos y más antiguos (normalmente hombres) y deja desamparados a los temporales (mayoritariamente, jóvenes y mujeres).

\section{Segregación horizontal y vertical en el mundo del trabajo}

Otro rasgo fundamental que sirve para entender el mundo del trabajo hoy en día es la existencia de las llamadas segregación horizontal y vertical. La segregación horizontal hace referencia al predominio de uno u otro sexo en una determinada profesión o actividad, mientras que la segregación vertical define las dificultades que encuentra uno de los sexos (las mujeres) para acceder a 
“Transfer” XV: 1-2 (2020), pp. 207-232. ISSN: 1886-554

las cúpulas directivas de las empresas o de las instituciones en que trabajan.

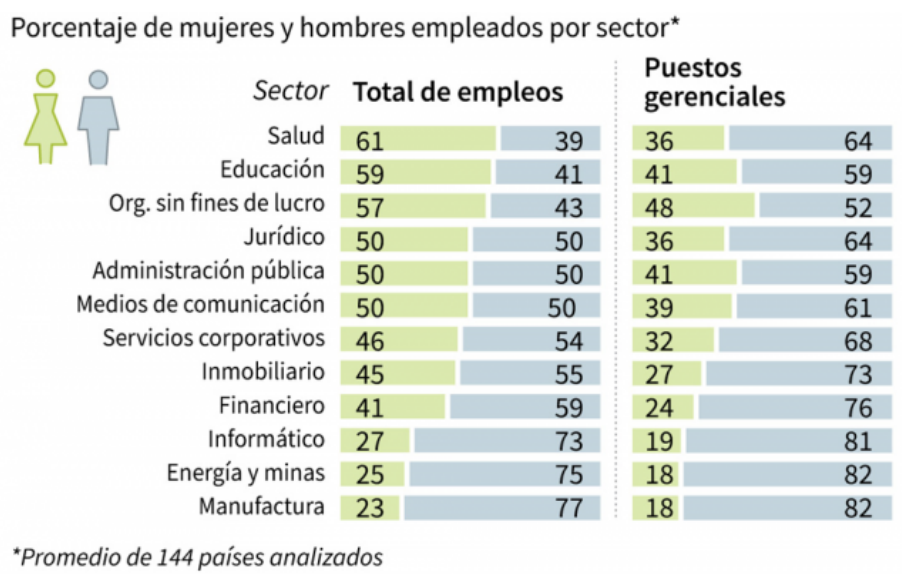

Fig. 5. Brecha de género en el trabajo. Fuente: Informe Global de la Brecha de Género 2017, Foro Económico Mundial.

En la Fig. 5 podemos observar ambos porcentajes. En cuanto a la segregación o discriminación horizontal, es de destacar, por un lado, el predominio de mujeres en ámbitos como la salud o la educación; y por otro lado, el predominio (todavía más acusado) de los hombres en sectores como la manufactura, energía y minas, informática, financiero, etc. con porcentajes que van desde el $23 \%$ hasta el 41\%. A menudo se considera esta segregación laboral por sexo uno de los fundamentos de lo que se llama techo de acero, es decir, una diferencia o brecha salarial entre hombres y mujeres. La Fig. 6, con datos procedentes de la Organización para la Cooperación y el Desarrollo Económicos (OCDE) para 2010, muestra la existencia de una asimetría entre los salarios de los hombres y los de las mujeres, que va desde el 6\% en España o Hungría hasta el $39 \%$ en Corea. 
“Transfer” XV: 1-2 (2020), pp. 207-232. ISSN: 1886-554

OECD nations' pay gap by gender (2010)

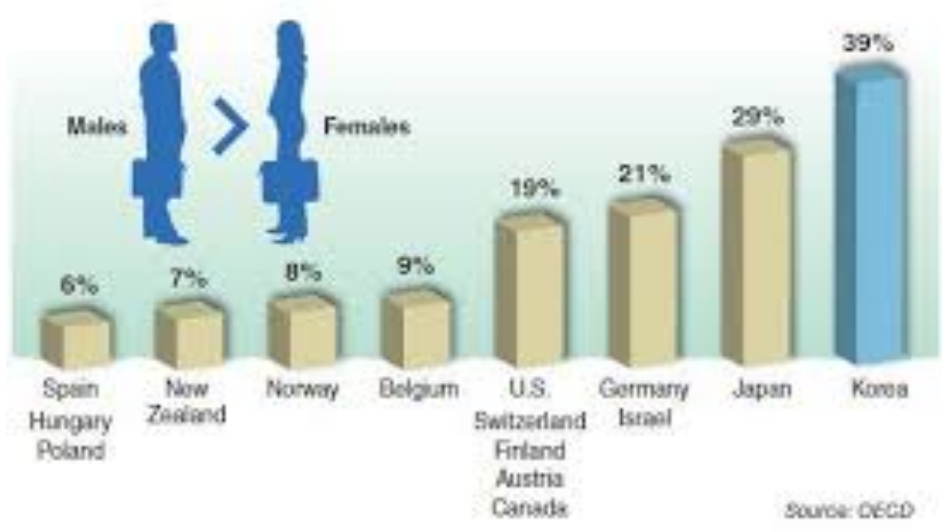

Fig. 6. Brecha salarial entre hombres y mujeres (2010). Fuente: OCDE.

La segregación horizontal, tanto de un sexo como de otro, es preocupante, sobre todo si se consolida a través de las décadas, pues supone perpetuar los estereotipos de qué profesiones son (o deben ser) femeninas y cuáles no. Si a ello le sumamos la Segregación vertical, convendremos entonces en que la situación es especialmente preocupante para las mujeres. Si volvemos a la Fig. 5 , comprobamos con preocupación que el llamado techo de cristal existe en todos los sectores laborales, incluso en aquellos que tradicionalmente convenimos en considerar feminizados, como la salud o la educación. Cifras desagregadas proporcionadas por la OCDE para el año 2009 (véase Fig. 7) sitúan el porcentaje de mujeres en puestos directivos en el conjunto de los países de la OCDE en torno al 10\%. La misma gráfica nos muestra también las enormes diferencias entre Noruega (auténtica pionera en la paridad en cargos directivos, con cerca de un $40 \%$ de mujeres en pues- 
“Transfer” XV: 1-2 (2020), pp. 207-232. ISSN: 1886-554

tos de responsabilidad) y el caso de Japón, en que apenas llega al $4 \%$.

\section{Existencia de una discriminación indirecta sistémica}

Un tercer rasgo, que se deriva de los dos anteriores, es la existencia de una discriminación indirecta persistente, de carácter sistémico. Ibáñez Pascual (2008: 118) apunta que "la segregación ocupacional también se suele asociar a la discriminación indirecta de las mujeres en los mercados laborales, desde el momento en que los trabajos donde la presencia femenina es mayoritaria reciben menores recompensas." La acción combinada de dos tipos de segregación (horizontal y vertical), de una cultura laboral claramente discriminatoria para las mujeres en general y de unos estereotipos y unas ideologías de género que todavía -y con demasiada frecuencia- separan lo femenino de lo profesional, sitúan a muchas mujeres en inferioridad de condiciones cuando intentan incorporarse al mercado de trabajo. En muchos casos, por desgracia, esta discriminación indirecta inicial se mantendrá a lo largo de toda su carrera profesional.

4. Asunción de las mujeres del grueso de las tareas domésticas. A los tres anteriores, añadiremos otro rasgo que, pese a los avances indudables en las últimas décadas, sigue dando lugar en muchos casos a una brecha importante: de manera genérica, las mujeres asumen el grueso de las tareas domésticas y del cuidado de los hijos o de los mayores, tareas tradicionalmente no remuneradas que, además, acarrean considerable esfuerzo y desgaste físicos. La diferencia, en los países desarrollados, sigue siendo muy importante, ya que las mujeres (como media estimada) dedican casi 2 horas al día más que los hombres a las tareas del hogar, que no tienen reconocimiento ni retribución alguna (véase Fig. 7). Pese a que la brecha va reduciéndose poco a poco, todavía persiste una ideología de género (cf. Cameron 2003, Talbot 2003) que asocia las tareas domésticas y los cuidados de los familiares a las mujeres. 
“Transfer” XV: 1-2 (2020), pp. 207-232. ISSN: 1886-554

Las consecuencias de estos rasgos, que hemos presentado de manera genérica, son bien conocidas. En primer lugar, los hombres obtienen, por norma, trabajos más cualificados (pese al menor nivel de formación con que partían). Ello trae como consecuencia que las mujeres, por tanto, tienen menores oportunidades laborales y son (como refleja la Fig. 3) las principales candidatas a la situación de desempleo. Acaban desempeñando, por tanto, empleos de peor calidad, fundamentalmente trabajos a tiempo parcial, cuando en realidad deseaban un empleo más estable.

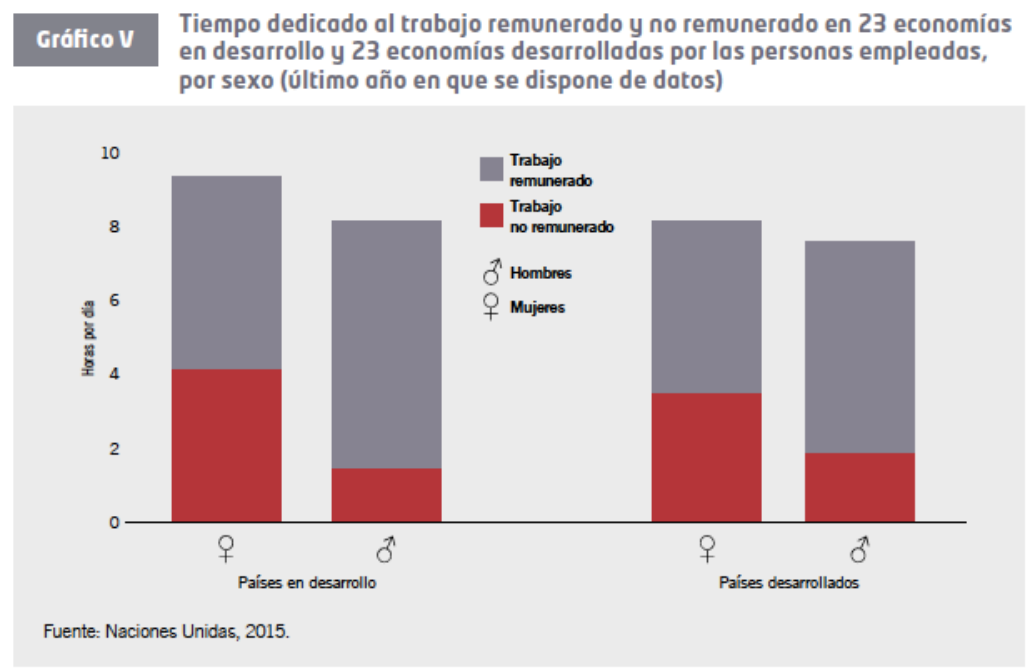

Fig. 7. Tiempo dedicado (por hombres y mujeres) al trabajo remunerado y no remunerado (Datos de 2015. Fuente: Naciones Unidas)

Todo ello provoca un fenómeno preocupante, muy extendido y que requiere una profunda reflexión: la sobrecualificación de las mujeres, que concurren al mercado laboral con una titulación 
“Transfer” XV: 1-2 (2020), pp. 207-232. ISSN: 1886-554

superior a la requerida y que, en una paradoja cruel, no obtienen el trabajo para el que tienen más méritos que sus colegas masculinos y, si lo obtienen, acaban recibiendo un menor salario. Esta perversión del mercado laboral empuja a muchas mujeres a disponer dos currículos, uno completo y otro con menos méritos de los que poseen, como forma de hallar mejor acomodo en las ofertas de trabajo existentes. Finalmente, no hace falta insistir en otro hecho bien conocido: resulta especialmente difícil para las mujeres conciliar la vida personal y la vida laboral. En definitiva, las mujeres, que son una mayoría demográfica, que poseen una formación apreciablemente superior a los hombres, que muestran una presencia creciente (quizás aún insuficiente) en el mercado laboral, no encuentran su sitio en él, pues se encuentran aprisionadas y relegadas en una cultura profesional que les es ajena y que les impide disponer de modelos de referencia.

\section{La traducción, una profesión feminizada: Pasado, presente y futuro de una paradoja}

1. La traducción editorial, ¿una profesión feminizada? Datos para un debate que no cesa

¿Qué ocurre, pues, en el campo de la traducción? ¿Posee esta profesión rasgos distintos al conjunto del mercado laboral, o comparte las mismas tendencias que hemos visto en los apartados anteriores?

A efectos oficiales, y según los datos actuales del INE (Instituto Nacional de Estadística), traductoras y traductores forman parte del grupo 2923 ("Filólogos, intérpretes y traductores"), como vemos en la Fig. 8: 
“Transfer” XV: 1-2 (2020), pp. 207-232. ISSN: 1886-554

\section{Filólogos, intérpretes y traductores}

29231010 Filólogos

29231038 Interpretes de lenguas de signos

29231056 Lingüistas.

29231074 Traductores-Interpretes.

29231029 Grafólogos

29231047 Interpretes en general.

29231065 Traductores, en general.

Fig. 8. Categoría profesional (según los datos oficiales del INE) en que se engloban traductores y traductoras

En los anuncios de trabajo, al especificar los requisitos de esta profesión, se exigen los siguientes conocimientos y habilidades, entre otros:

- conocimientos de lengua y literatura;

- capacidad de expresión oral y escrita;

- $\quad$ análisis de textos;

- uso de diccionarios, glosarios y repertorios;

- traducción de textos;

- interpretación oral (tanto simultánea como consecutiva);

- uso de herramientas de traducción asistida;

- manejo de memorias de traducción;

- uso y elaboración de terminología especializada;

- corrección y edición de textos;

- planificación lingüística;

- etc.

Además de estos requisitos de carácter técnico, es de destacar que no se suele aplicar una exigencia que es harto habitual en la mayoría de profesiones: la edad o el sexo de candidatos o candidatas. Una interpretación que se ha barajado con frecuencia es que esto indica que en la profesión de traductor/a existe un menor grado de discriminación por razones de edad o sexo. Otras interpretaciones apuntan a la metáfora recurrente de la 'invisibilidad' (véase Venuti 2008) que viene persiguiendo a esta profesión desde hace siglos. ¿Tan poco trascendentes son los traductores que no importa quién haga el trabajo? 
“Transfer” XV: 1-2 (2020), pp. 207-232. ISSN: 1886-554

Si analizamos con más detalle la traducción editorial en el estado español, nos daremos cuenta que ya en un informe de 2001 (véase Ministerio de Cultura 2001) las mujeres mostraban una mayor tendencia a dedicarse a la traducción de manera exclusiva: en concreto, un $49 \%$ de las mujeres, por un $37 \%$ de los hombres. Un informe de 2010 (véase ACE Traductores 2010) aportaba el dato de que, las mujeres, como colectivo, eran mayoría (54\%) en el mundo de la traducción editorial, frente al 45\% de hombres. Un informe de ese mismo año (Ministerio de Cultura 2010) define con más precisión el perfil profesional en el mundo de la traducción:

- se constata un aumento progresivo del número de mujeres $(54,1 \%)$.

- se confirma el dato, muy relevante, de que los traductores intensivos suelen ser mujeres (60\%).

- se apunta que, en el mundo de la traducción editorial, las mujeres tienen unos ingresos más elevados.

El último informe publicado (ACE Traductores 2017) confirma, en líneas generales, los datos anteriores, y añade un dato sobre hábitos lectores que puede tener su importancia para definir la profesión de traductor/a: en general, las mujeres leen más que los hombres (salvo en el caso de lectura por profesión o estudios). Fruela Fernández (2012) considera así la traducción editorial:

En la actualidad, por tanto, la traducción de libros continúa la tendencia social e histórica y se muestra como un sector laboral feminizado, ya que las mujeres se orientan a él antes que a otros -como demuestra su mayor presencia frente al conjunto del mercado- y con mayor dedicación, frente a la tendencia de los hombres a simultanear varias profesiones. (Fernández 2012: 52)

Que la traducción constituye un sector feminizado no es, en realidad, ninguna novedad. Desde la Edad Media y, sobre todo, el Renacimiento, la traducción ha constituido una de las pocas salidas, socialmente aceptadas, para la creatividad femenina, una 
“Transfer” XV: 1-2 (2020), pp. 207-232. ISSN: 1886-554

opción profesional que no cuestionaba la superioridad creadora del hombre pero que, al mismo tiempo, ha servido para iniciar/ fundar una genealogía femenina que los estudios de género y traducción están poniendo en valor durante las últimas décadas (véase Godayol 2000, Delisle 2002).

La existencia de muchos casos bien documentados históricamente (Marie de France, Margaret Roper More, Mary Sidney Herbert, Aphra Behn, María Rosario Romero, Inés de Joyes y Blake, etc.), y de muchos otros de los que no se conserva memoria, ha puesto de relieve un hilo conductor repetidamente negado por la traductología hasta hace unas pocas décadas: la especial relación de las mujeres, de muchas mujeres, con la lengua, la cultura, el conocimiento y la capacidad de expresión artística (véase Godayol 2000, Delisle 2002, Bolufer 2017).

¿Qué significa, por tanto, que la traducción sea un ámbito feminizado? La consideración habitual es meramente cuantitativa: hay más traductoras que traductores en el mundo editorial. En este sentido, todos los datos de que disponemos, relativos a la traducción editorial, confirman la feminización de esta actividad.

Podemos, también, interpretarlo de otra manera: se observa una sexualización de la profesión, una atribución de rasgos de género a una profesión. En este segundo planteamiento, la traducción es mujer. Es decir, los contenidos, los procedimientos y las habilidades de la profesión de traductor/a se asocian a las mujeres y a los estereotipos sexuales que se identifican con las mujeres. Ello, por desgracia, está asociado a connotaciones negativas. Chamberlain (1988) denuncia una tradición de metaforización discriminatoria en las teorías de la traducción, en la línea de les belles infidèles. Teóricos como John Florio, Friedrich Schleiermacher o George Steiner sexualizan la traducción en tanto que mujer, ambos como espacio de lo secundario, sometidos ambos al hombre, a su sexualidad y a su creatividad (el hombre queda equiparado a la escritura original). Aportaciones más recientes desde los estudios de género, feminismo y traducción están generando relecturas positivas de la especial relación entre mujer y traducción, como espacios de creatividad y reescritura, al tiempo que nuevas metáfo- 
“Transfer” XV: 1-2 (2020), pp. 207-232. ISSN: 1886-554

ras, como Pandora, la frontera, o metramorfosis (Littau 2000, Godayol 2000, Shread, von Flotow 2008).

Para Bourdieu el orden simbólico masculino se impone, de manera generalizada, en todas las actividades y espacios:

\begin{abstract}
El orden social funciona como una inmensa máquina simbólica que tiende a ratificar la dominación masculina en la que se apoya: es la división sexual del trabajo, distribución muy estricta de las actividades asignadas a cada uno de los dos sexos, de su espacio, su momento, sus instrumentos; [...] (Bourdieu 2000: 22).
\end{abstract}

Richard Anker, en su libro Gender and Jobs (1998), señala las creencias estereotipadas acerca de las mujeres, que divide en positivas y negativas. La siguiente tabla es un resumen aproximado de los rasgos que, de manera prototípica, se consideran femeninos:

\title{
Estereotipos positivos $\mid$ Estereotipos negativos
}

Tendencia a cuidar a las personas Menor tendencia a dirigir o dar Habilidad y experiencia en tareas domésticas

Mayor habilidad manual Mayor honradez Apariencia física Mayor disposición a recibir órdenes Mayor docilidad Menor tendencia a afiliarse a órdenes Menor fuerza física

Menor aptitud para matemáticas o ciencias

Menor disposición a viajar Menor disposición al trabajo físico Menor disposición al uso de la sindicatos

Aceptación de trabajo monótono fuerza

Mayor interés en trabajar en casa

Fig. 9. Estereotipos positivos y negativos sobre las mujeres (Adaptado de Anker 1998: 24-27)

¿Cuáles son las consecuencias de esta división sexual del trabajo? Las indicamos a continuación, según Anker (1998), en una formulación sucinta: 
“Transfer” XV: 1-2 (2020), pp. 207-232. ISSN: 1886-554

1. Afecta negativamente a la imagen que los hombres tienen de las mujeres y las que éstas tienen de sí mismas;

2. Afecta negativamente a la eficiencia y el funcionamiento del mercado laboral;

3. Confiere gran rigidez al mercado laboral;

4. Afecta negativamente a la educación y formación de futuras generaciones;

5. Expulsa a muchas mujeres del mercado laboral;

6. Provoca diferencias en los salarios, en detrimento de las mujeres;

7. Contribuye a la pobreza y desigualdad social.

Atendiendo tanto a los datos estadísticos proporcionados en este apartado como a los estereotipos definidos por Anker (1998), y aun otros que atribuyen a las mujeres una mayor competencia lingüística o cultural (Talbot 2003), habremos de concluir que la traducción es una actividad eminentemente femenina. En 3.2 haremos una breve incursión para analizar quién se dedica a la traducción editorial, centrándonos en los Premios Nacionales de Traducción.

\section{Pero ... ¿quién se dedica a la traducción editorial? Los Premios} Nacionales de Traducción

En 3.1 reflejábamos la mayor presencia femenina, y su mayor implicación, en la traducción editorial, según todos los informes consultados. Sin embargo, la división sexual identificada por Bourdieu (2000) provoca que las mujeres, pese a su mayoría estadística y a los rasgos femeninos del mundo profesional de la traducción, poseen todavía una menor visibilidad y reconocimiento. Veamos, si no, los ejemplos de los dos premios estatales de traducción, concedidos anualmente por el Ministerio de Cultura:

- el Premio Nacional a la Mejor Traducción (concedido desde 1984, heredero del Premio Fray Luis de León, que se instauró en 1956); y 
“Transfer” XV: 1-2 (2020), pp. 207-232. ISSN: 1886-554

- el Premio Nacional a la Obra de un Traductor (concedido desde 1989, como reconocimiento a la trayectoria de un/a traductor/a).

La nómina de premiados y premiadas ofrece un buen diagnóstico de los desequilibrios y sesgos de género, prestigio, canon literario, ámbito lingüístico y cultural, comunidad nacional, etc. Con referencia al conjunto de ediciones del Premio Nacional a la Mejor Traducción, vemos (Tabla 2) la brecha enorme que separa a los hombres premiados (36) de las mujeres premiadas (12). Vemos también cómo, en un (apresurado) intento reciente de compensación, desde 2011 un total de 7 mujeres han recibido el premio: Olivia de Miguel (2011), Luz Gómez García (2012), Carmen Montes Cano (2013), Eva Almazán y María Alonso Seisdedos (2014), Ana María Bejarano Escanilla (2016) y Neila García Salgado (2018). La estadística anterior a 2011, cuando menos, francamente atentatoria contra una profesión feminizada, reflejo sin duda de un orden simbólico masculino. ${ }^{4}$

\begin{tabular}{|l|c|c|c|c|}
\hline & Español & Catalán & Gallego & Euskera \\
\hline Hombres & 28 & 4 & 4 & 0 \\
\hline Mujeres & 10 & 0 & 2 & 0 \\
\hline
\end{tabular}

Tabla 2. Galardonados con el Premio Nacional a la Mejor Traducción (1984-2018). Fuente: Ministerio de Cultura.

Somos, también, conscientes de otras discriminaciones, como las lingüísticas o culturales: 38 premiados/as traducen des-de una lengua extranjera al español, 6 al gallego (aunque 4 de ellos/as en el premio otorgado en 2014 por la traducción de Ulises de James Joyce), 4 en catalán y no hay premiados/as por traduccines al euskera. Sigue pendiente el reconocimiento de la realidad pluri-

${ }^{4}$ Los datos proceden de la página oficial del Ministerio de Cultura y Deportes (Disponible en: <<https://bit.ly/2XsRbRE $>>$, Última consulta: 14 de marzo de 2019). 
“Transfer” XV: 1-2 (2020), pp. 207-232. ISSN: 1886-554

nacional y plurilingüística del estado español que -paradójicamente- la propia traducción encarna y proclama.

Otro aspecto significativo es el tipo de textos que traducen los traductores y traductoras que han merecido el Premio Nacional a la Mejor Traducción. Según Fernández:

los traductores tienden a orientarse hacia los géneros de mayor capital simbólico, aquellos más "formales" y "nobles", mientras que las traductoras hallan más espacio entre los géneros de mayor rendimiento comercial y, por lo general, menos legitimados literariamente. (Fernández 2014: 54)

Los datos extraídos de la página oficial del Ministerio de Cultura y Deportes no hacen sino confirmar esta tendencia. Los traductores premiados lo han sido, en gran parte, por sus versiones de un buen número de obras clásicas: Las mil y una noches, las Sátiras de Horacio, el Corán y la Biblia, así como obras de autores tan prestigiosos como Shakespeare, Dante, Ariosto, Lucano, James Boswell, Benito Arias Montano, Tito Lucrecio Caro, etc. A ello se suman obras de autores prestigiosos del siglo XX (James Joyce, Paul Celan) y hasta obras procedentes de culturas exóticas (poesía nórdica). Por contra, las traductoras premiadas apenas incluyen autores clásicos (Francis Bacon, James Joyce), junto a otros menos conocidos del siglo XX (Marianne Moore, David Grossman, A.S. Byatt, etc.).

Además, los traductores lo hacen desde un amplio abanico de lenguas, que incluyen tanto lenguas clásicas (griego y latín) hasta lenguas modernas europeas (inglés, alemán, portugués, italiano, albanés) y grandes lenguas internacionales (árabe, chino). Las tra-ductoras, en consonancia con su escaso número, traducen desde un número reducido de lenguas (inglés, sueco, serbio, hebreo, griego moderno, árabe). 
“Transfer” XV: 1-2 (2020), pp. 207-232. ISSN: 1886-554

Por lo que respecta al Premio Nacional a la Obra de un Traductor, se observan similares tendencias, con 23 hombres premiados y 7 mujeres premiadas (véase Tabla 3). ${ }^{5}$

\begin{tabular}{|l|c|c|c|c|}
\hline & Español & Catalán & Gallego & Euskera \\
\hline Hombres & 21 & 1 & 0 & 1 \\
\hline Mujeres & 6 & 1 & 0 & 0 \\
\hline
\end{tabular}

Tabla 3. Galardonados con el Premio Nacional a la Obra de un Traductor (1989-2018). Fuente: Ministerio de Cultura.

El desequilibrio entre espacios lingüístico-culturales es, si cabe, más acusado que en el Premio Nacional a la Mejor Traducción. De las 30 ediciones hasta la fecha, 27 premios reconocen las traducciones al castellano, mientras que el resto son cifras absolutamente ridículas: 2 premios han ido a traductores (un hombre y una mujer) al catalán, 1 al euskera (un hombre), y ni un solo premio a traductores/as al gallego. Vemos cómo, en conjunto, ambos Premios Nacionales a la traducción editorial perpetúan una doble subordinación histórica: la de las traductoras (mayoritarias, recordemos, en la profesión) y la de los ámbitos lingüísticoculturales (supremacía excesiva de una lengua, el castellano, a expensas de las otras lenguas del estado).

Retornemos a la pregunta inicial: ¿es la traducción una profesión feminizada? Sabemos que una pregunta con esta formulación es, en sí, irresoluble, pero la hemos planteado para avivar un debate que sigue siendo paradójico. Creíamos que los datos contenidos en 3.1, sobre la traducción editorial, resolvían la cuestión, pero no hacen sino acrecentar la paradoja en que llevamos embarcados desde hace siglos. La mujer encarna (y representa) a la traducción, pero los hombres traducen mejor, o al

\footnotetext{
${ }^{5}$ Datos extraídos de la página oficial del Ministerio de Cultura y Deportes (Disponible en: <<https://bit.ly/2ZWxBz5 >>, Última consulta: 14 de marzo de 2019).
} 
“Transfer” XV: 1-2 (2020), pp. 207-232. ISSN: 1886-554

menos reciben los premios y galardones. Hay más traductoras editoriales, pero los más conocidos son hombres (Miguel Sáenz, Agustín García Calvo, Valentín García Yebra, etc.).

\title{
Otra vuelta de tuerca: Mujer y traducción, ámbitos de lucha y resistencia
}

Clare Walsh (2001) apunta:

\begin{abstract}
one effect of women's entry into traditionally male-dominated domains has been to make explicit the implicitly masculinist nature of the beliefs, norms, values and practices that masquerade as gender-neutral professional norms within these communities of practice. (Walsh 2001: 20)
\end{abstract}

En el mundo de la traducción, este vaticinio resulta aún prematuro. Pocos pueden dudar que, hoy en día, la traducción es una profesión -o tarea, o actividad, o anhelo, o arte- feminizada. Todo apunta a ello: los datos estadísticos generales, los porcentajes de estudiantes en la universidad, su nivel de formación. De ellas son también la formación en lenguas extranjeras, la capacidad expresiva y comunicativa, la especial aptitud para la mediación y las relaciones interpersonales, entre otros rasgos predominantes. Pese a ello, parece que todavía es pronto para revertir los vientos de la tradición. La traducción, en la medida en que es percibida como actividad privada, es y ha sido un refugio para las mujeres a lo largo de la historia. No obstante, en la medida en que tiene una dimensión pública (sobre todo la traducción editorial), "is in danger of becoming an asymmetrical two-tier system in which women's subordinate status is institutionalized" (Walsh 2001: 2).

Desde estas páginas invitamos a transformar una actividad feminizada como la traducción (y, por tanto, devaluada en la esfera pública), en espacio de subversión y transformación positiva. Se trata, fundamentalmente, de reconocer el potencial que tienen las mujeres para crear con la palabra y con la comunicación. La 
“Transfer” XV: 1-2 (2020), pp. 207-232. ISSN: 1886-554

traducción es una práctica (y un discurso) que tiene la virtud de poder convertir los textos (y los usuarios de dichos textos) en agentes de poder. Puede, también, generar espacios de resistencia, de visibilidad, de originalidad, de lucha por valores como la igualdad o la solidaridad (véase Santaemilia 2017). Queda pendiente aún (pues es, quizá, irresoluble) la cuestión de la (in)visibilidad de traductores y traductoras, que cuenta por igual con defensores y detractores, pero que no debe esconder el necesario reconocimiento económico y profesional de quienes se dedican a esta (in)grata tarea. Cumple igualmente a los traductores, presentes o futuros, y quizá más a las traductoras, explicar cuál es el valor social de la traducción, sus dificultades, su relevancia como instrumento comunicativo e ideológico, etc. Se impone una alianza (conceptual, estratégica, etc.) entre género, feminismo y traducción, como han defendido numerosas voces desde Godard (1989) hasta Castro \& Ergun (2017). Las tres son fuerzas renovadoras y críticas con todo lo existente. La traducción es una labor de (re)creación, y no una mera tarea reproductiva, un espacio que hay que ganar metro a metro. Un espacio de convicciones ideológicas y de posturas éticas, pero también un espacio para el placer, ya sea lingüístico, cultural o sexual, afectivo, sentimental o evocativo.

\section{Referencias bibliográficas}

ACE TRADUCTORES. (2010). Libro Blanco de la traducción editorial en España. Madrid: Ministerio de Cultura.

---. (2017). Informe del valor económico de la traducción editorial.

Madrid: Ministerio de Educación, Cultura y Deporte.

ANKER, Richard. (1998). Gender and jobs. Sex segregation of occupations in the world. Ginebra: International Labour Office.

BOLUFER, Mónica. (2017). "La traducción como práctica cultural: agentes y contextos. A propósito de tres traductoras en la España del siglo XVIII". En: KEILHAUER, Annette \& Andrea PAGNI (eds.) Refracciones. Traducción y género en las 
“Transfer” XV: 1-2 (2020), pp. 207-232. ISSN: 1886-554

literaturas románicas / Réfractions. Traduction et genre dans les littératures romanes. Wien: Lit Verlag, pp. 23-40.

BOURDIEU, Pierre. (2000). La dominación masculina. Barcelona: Anagrama. Trad. Joaquín Jordá.

CAMERON, Deborah. (2003) "Gender and language ideologies". En : HOLMES, Janet \& Miriam MEYERHOFF (eds.). Handbook of Gender and Language Research. Oxford: Blackwell, pp. 44767.

CASTRO, Olga \& Emek ERGUN (eds.). (2017). Feminist Translation Studies: Local and Transnational Perspectives. Londres-Nueva York: Routledge.

CHAMBERLAIN, Lori. (1992 [1988]). "Gender and the metaphorics of translation." Signs, 13.3: 454-472.

DELISLE, Jean. (ed.). (2002 [1999]). Portraits de traductrices. Ottawa: Artois Presses Université.

FERNÁNDEZ, Fruela. (2014), “Assessing masculine domination in a cultural field: women translators and book translation awards in Spain (1984-2012)". The Translator, 20(2): 162-177.

GODARD, Barbara. (1989). "Theorizing Feminist Discourse/ Translation". Tessera, 6: 42-53.

GODAYOL, Pilar. (2000). Espais de frontera: Gènere i traducció. Vic: Eumo Editorial.

IBÁÑEZ PASCUAL, Marta. (2008). "La segregación ocupacional por sexo a examen. Características personales, de los puestos y de las empresas asociadas a las ocupaciones masculinas y femeninas". Revista Española de Investigaciones Sociológicas (Reis), 123: 87-122.

LITTAU, Karin. (2000). “Pandora's Tongues”. TTR - Traduction, Terminologie, Rédaction, 13(1): 21-35.

MINISTERIO DE ASUNTOS EXTERIORES Y COOPERACIÓN. (2012). Libro Blanco de la traducción y la traducción institucional. Madrid: Ministerio de Asuntos Exteriores y Cooperación.

MINISTERIO DE CULTURA. (2001). La traducción editorial en España. Madrid: Centro de Documentación del Libro y la Lectura, D.G. del Libro, Archivos y Bibliotecas. 
“Transfer” XV: 1-2 (2020), pp. 207-232. ISSN: 1886-554

--- (2010). La traducción editorial en España. Madrid: Servicio de Estudios y Documentación, S.G. de Promoción del Libro, la Lectura y las Letras Españolas, D.G. del Libro, Archivos y Bibliotecas.

PEÑA, Daniel. (2010). “Cien años con mujeres en la universidad”. El País, 8-03-2010 . Disponible en:

$<<$ https://elpais.com/sociedad/2010/03/08/ actualidad/1268002812_850215.html

SANTAEMILIA, José. (ed.). (2017). Traducir para la igualdad sexual / Translating for Sexual Equality. Granada: Editorial Comares.

SILIÓ, Elisa. (2018) "Pocas rectoras para una mayoría de universotarias". El País, 5-03-2018. Disponible en: $<<$ https://elpais.com/politica/2018/03/04/ actualidad/1520195159_462681.html

SHREAD, Carolyn. (2007). "Metamorphosis or Metramorphosis? Towards a feminist ethics of difference in translation". TTR, 20(2): 213-242.

TALBOT, Mary M. (2003). “'Women Rule as a Natter of Fact': Reproducing and Challenging Gender Stereotypes". En: SANTAEMILIA, José. (ed.) Género, lenguaje y traducción. Valencia: Universitat de València / Dirección General de la Mujer, pp. 26-41.

TOBARRA, Sebastián. (2010). "Más formadas, pero con peores empleos". El País, 14-11-2010. Disponible en: $<<$ https://elpais.com/diario/2010/11/14/ sociedad/1289689201_850215.html

VENUTI, Lawrence. (2008). The Translator's Invisibility: A history of translation. Londres/Nueva York: Routledge. $2^{\text {nd }}$ ed.

VON FLOTOW, Luise. (2008). "Postface: Contested Gender in Translation: Intersectionality and Metramorphics". Palimpsestes, 22 (« Traduire le genre: femmes en traduction»): 245255.

WALSH, Clare. (2001). Gender and Discourse: language and power in politics, the church and organisations. Londres: Longman. 
“Transfer” XV: 1-2 (2020), pp. 207-232. ISSN: 1886-554

Fecha de recepción: 2.05.2019 Fecha de aceptación: 01.06.2019

\section{Resumen:}

Este artículo aportará datos para un debate sobre la presencia de las mujeres en el mundo de la traducción profesional, sobre todo en la traducción literaria o editorial. Para ello, observaremos qué presencia tienen las mujeres en la universidad y en el mercado laboral, atendiendo a las diversas disciplinas y ámbitos profesionales. Realizaremos un diagnóstico de la situación de las mujeres en el mundo profesional (prestando atención a la cultura laboral masculinizada, los estereotipos y expectativas sociales en torno a las mujeres, las cualidades 'femeninas', etc.), lo que nos llevará a concluir que los puestos de trabajo están sexuados en su práctica totalidad, y que las mujeres sufren habitualmente una doble segregación (horizontal y vertical) que limita seriamente su participación plena en el mundo laboral. A raíz del diagnóstico realizado, pasaremos a valorar las consecuencias que todo ello tiene para las mujeres. Tras repasar algunos indicadores contenidos en diversos informes sobre traducción editorial (2010, 2012, 2017), publicados tanto por el Ministerio de Cultura como por la asociación ACE Traductores, que ponen de manifiesto que la traducción es un sector laboral feminizado, reflexionamos (de la mano de autores como Pierre Bourdieu 1998, Richard Anker 1998 o Clare Walsh 2001, sobre los estereotipos (positivos y negativos) tradicionalmente asociados a las mujeres, y su concreción en el mundo de la traducción.

Palabras clave: Traducción como profesión, Feminización, Traducción editorial, Igualdad sexual. 
“Transfer” XV: 1-2 (2020), pp. 207-232. ISSN: 1886-554

TRANSLATING TODAY: A PORTRAIT OF A FEMINIZED

PROFESSION. SOME ETHICAL AND WORK-RELATED ISSUES

\begin{abstract}
:
This paper offers data for a debate on the presence of women in the world of professional translation, especially in literary or editorial translation. In order to do so, statistics will be given on the percentages of women at university and as professionals, according to a variety of study fields and jobs. A diagnosis will be offered on the situation of women in the job market, emphasizing issues such as a masculinized work culture, women-related social expectations, 'feminine' qualities, and so on. This will lead us to conclude that job positions are on the whole sex-segregated, with women traditionally suffering from both horizontal and vertical discrimination, which seriously restricts their full participation in the job market. The ensuing consequences for women will be assessed. After going through a number of institutional reports on editorial translation $(2010,2012,2017)$ published both by the Spanish Ministry for Culture and by the ACE Traductores Association, and which clearly point to translation as a feminized job sector, some reflection (following Bourdieu 1998, Anker 1998 or Walsh 2001) is given on the (positive and negative) stereotypes associated with women, and particularly in the translating profession.
\end{abstract}

Keywords: Translating Profession, Feminization, Editorial Translation, Sexual Equality. 